\title{
Optical fiber refractometers with response in the visible spectral region by means of ITO coatings
}

\section{Refractómetros de fibra óptica con respuesta en el espectro visible mediante la utilización de recubrimientos de ITO}

\author{
S. López, C. R. Zamarreño ${ }^{(*)}$, M. Hernaez, I. Del Villar, I. R. Matias, F. J. Arregui \\ Electrical and Electronic Engineering Department, Public University of Navarra, Pamplona Spain. \\ (*) Email: carlos.ruiz@unavarra.es \\ Recibido / Received: 06/09/2011. Revisado / Revised: 21/12/2011. Aceptado / Accepted: 13/01/2012.
}

\begin{abstract}
:
This work presents the fabrication of optical fiber refractometers based on indium tin oxide (ITO) coatings with response in the visible spectral region. ITO thin-films have been sputtered by employing a rotating mechanism that enables the fabrication of smooth and homogeneous coatings onto the optical fiber core. Fabricated ITO coated optical fiber devices present several resonances in the visible and near infrared region. These resonances show high optical power attenuations (more than $10 \mathrm{~dB}$ ) in the visible spectral region, which produces changes in the colour of the output visible light. Therefore, since these resonances shift as a function of the refractive index (RI) of the surrounding medium it is feasible to determine the RI of the outer medium in contact with the ITO coating by simply monitoring the chromatic coordinates (colour change), $x$ and $y$, of the visible output light.
\end{abstract}

Key words: Optical Fiber, Refractometer, Indium Tin Oxide, Resonances, Refractive Index, Sensor, Colour.

\section{RESUMEN:}

En este trabajo se presenta la fabricación de refractómetros de fibra óptica basados en recubrimientos de óxido de indio dopado con estaño (ITO) con respuesta en el visible. Para la fabricación de los recubrimientos se ha utilizado la técnica de sputtering combinada con un mecanismo de rotación que permite la fabricación de superficies homogéneas y poco rugosas sobre el núcleo de la fibra óptica. Los dispositivos fabricados muestran resonancias en la región del visible e infrarrojo cercano. Estas resonancias presentan atenuaciones altas de potencia (mayores de $10 \mathrm{~dB}$ ) en la región del visible lo que origina un cambio cromático la luz después de atravesar la región sensible. A su vez, el desplazamiento de las resonancias con el índice del medio externo en contacto con el ITO permite la determinación del índice de refracción mediante la monitorización de las coordenadas cromáticas (cambio de color), $x$ e $y$, de la luz visible a la salida de la fibra.

Palabras clave: Fibra Óptica, Refractómetro, Óxido de Indio Dopado con Estaño, Resonancias, Índice de Refracción, Sensor, Color.

\section{REFERENCIAS Y ENLACES / REFERENCES AND LINKS}

[1]. C. R. Zamarreño, M. Hernaez, I. Del Villar, F. J. Arregui, I. R. Matias, "ITO coated optical fiber refractometers based on resonances in the infrared region", IEEE Sens. J. 10, 365-366 (2010).

[2]. M. Hernaez, I. Del Villar, C. R. Zamarreño, F. J. Arregui, I. R. Matias, "Optical fiber refractometers based on lossy mode resonances supported by $\mathrm{TiO}_{2}$ coatings", Appl. Opt. 49, 3980-3985 (2010).

[3]. C. R. Zamarreño, P. Sanchez, M. Hernaez, I. Del Villar, C. Fernandez-Valdivielso, F. J. Arregui, I. R. Matias, "Dual-peak resonance-based optical fiber refractometers", IEEE Photonic. Tech. L. 22, 17781780 (2010). 
[4]. C. R. Zamarreño, P. Sanchez, M. Hernaez, I. Del Villar, C. Fernandez Valdivielso, F. J. Arregui, I. R. Matias, "LMR-based optical fiber refractometers based on transparent conducting and semiconducting oxide coatings: A comparative study", Proc. SPIE 7839, 78391E (2010).

[5]. C. R. Zamarreño, M. Hernaez, I. Del Villar, I. R. Matias, F. J. Arregui, "Thin-Film resonance supporting coatings deposited onto optical waveguides towards the fabrication of sensing devices", Recent Pat. Mater. Sci. 4, 28-34 (2011).

[6]. G. Q. Liu, F. L. Tang, L. Li, L. X. Gong, Z. Q. Ye, “Concentration detection of quantum dots in the visible and near-infrarred range based on surface plasmon resonance sensor", Mater. Lett. 65, 1998-2000 (2011).

[7]. I. del Villar, C. R. Zamarreño, M. Hernaez, I. R. Matias, F. J. Arregui, "Lossy mode resonance generation with indium tin oxide coated optical fibers for sensing applications," J. Lightwave. Technol. 28, 111117 (2010).

[8] I. Del Villar, C. R. Zamarreño, P. Sanchez, M. Hernaez, C. Fernandez-Valdivielso, F. J. Arregui, I. R. Matias, "Generation of lossy mode resonances by deposition of high-refractive-index coatings on uncladded multimode optical fibers", J. Opt. 12, 095503 (2010).

[9]. I. Kasik, J. Mrazek, O. Podrazky, M. Seidl, J. Aubrecht, P. Tobiska, M. Pospisilova, V Matejec, B. Kovacs, A. Markovics, M. Szili, "Fiber-optic detection of chlorine in water", Sensor. Actuat. B - Chem. 139, 139142 (2009).

[10]. P. R. Cooper, "Refractive index measurements of liquids used in conjunction with optical fiber", Appl. opt. 22, 3070-3072 (1983).

\section{Introduction}

Recently has been shown the fabrication of optical fiber refractometers based on metal oxides with characteristic optical and electrical properties, such as indium tin oxide (ITO), titanium dioxide $\mathrm{TiO}_{2}$ or indium oxide $\mathrm{In}_{2} \mathrm{O}_{3}$ [1-3]. These refractometers are based on a type of resonances that can be related with lossy modes and commonly are known as lossy mode resonances (LMRs), which have been described in several works $[4,5]$. LMRs, unlike the more familiar surface plasmon resonance (SPR) [6], have the advantage of being generated by both, TE and TM, light polarizations as well as allow the generation of more than one resonance without modifying the morphology of the fiber [7].

However, in most cases, the fabrication processes of the LMR supporting coatings, such as dip-coating or Layer-by-Layer, are time consuming or require the precise control of external parameters (pressure, humidity, temperature, etc.) to produce homogeneous and repetitive coatings [1-3,7-8]. In order to solve the problems mentioned above here it is presented an alternative approach. This approach basically consists of the application of the sputtering technique in order to produce thin and homogeneous ITO coatings around the core of optical fibers, which act as LMR supporting coatings with high attenuation bands. Here, the precise control of the fabrication conditions provided by the sputtering technique enables to determine the resonance characteristics with high precision and guarantee the repeatability of the experiments.

\section{Experimental process}

\section{2.a. Fabrication of ITO coatings}

For the fabrication of different devices fabricated in this study was used an optical fiber (FT200EMT from Thorlabs Inc.) with diameters of 200 and $225 \mu \mathrm{m}$ for the core and cladding respectively and numerical aperture 0.39 . Previously to the manufacture of ITO coating it is necessary to prepare the fiber using the procedure described in [7]. This process basically involves the removal of the cladding of plastic fiber along a length of $10 \mathrm{~cm}$ followed by a cleaning process using ultrasound. The process of preparation of the fiber described above will enable the deposition of the coating directly onto the fiber core. For the manufacture of ITO coatings it is used a sputtering equipment (K675XD from Quorum Technologies Ltd.) with a partial pressure of Argon between $6 \times 10^{-3}$ and 


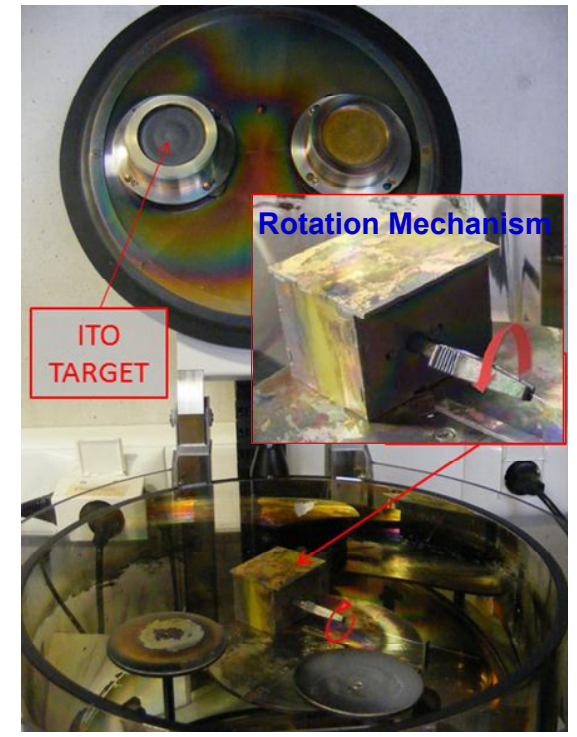

Fig. 1: Rotation mechanism introduced into the sputtering vacuum chamber for the deposition of ITO over the optical fiber.

$9 \times 10^{-3}$ mbar and intensity of $150 \mathrm{~mA}$ which allowed to achieve deposition rates of between 0.2 and $0.4 \mathrm{~nm} / \mathrm{sec}$.

Sputtering technique is mainly intended to be used with planar substrates which meant that it was necessary to implement a new mechanism in order to rotate the optical fiber during the deposition process. The rotation device was based on a speed controlled DC motor and incorporates a system to hold the fiber parallel to the deposition plane as it is represented in Fig. 1. After the ITO coating fabrication, the fiber was subjected to a thermal treatment at $250^{\circ} \mathrm{C}$ in vacuum for two hours in order to improve the properties of the coating [9].

\section{2.b. Characterization of the fiber optic refractometers}

After the ITO coating deposition onto the optical fiber, a portion of $4 \mathrm{~cm}$ approximately was cleaved (LCD-200 Vytran Inc.) and spliced (FITEL S176, Furukawa Co. Ltd.) on both ends to $200 \mu \mathrm{m}$ core diameter optical fiber pigtails. One of the ends was connected to a white light source (halogen lamp, model ASBN-W-150-H from Spectral Products) and the other one was attached to the single end of a $200 \mu \mathrm{m}$ bifurcated optical fiber (VIS/NIR, from Oceanoptics Inc.) as shown in Fig. 2. The other end of the bifurcated optical fiber was connected to two spectrometers (HR4000 and NIR-512, both from Oceanoptics Inc.) in order to cover a larger spectral range (400 $\mathrm{nm}$ to $1700 \mathrm{~nm})$. The spectral data were obtained at the distal end of the fiber once the light has passed through the sensitive region.

The response of the device was recorded when the sensitive region (see Fig. 2) was subjected to external medium refractive index (EMRI) variations. EMRI varied from air $(n=1)$ to water $(n=1.333)$ and $80 \%$ glycerin/water $(n=1.447)$. Refractive indices were measured at $590 \mathrm{~nm}$ [10]. All the measurements were performed at room temperature $\left(20^{\circ} \mathrm{C}\right.$ and $30 \%$ $\mathrm{RH})$.
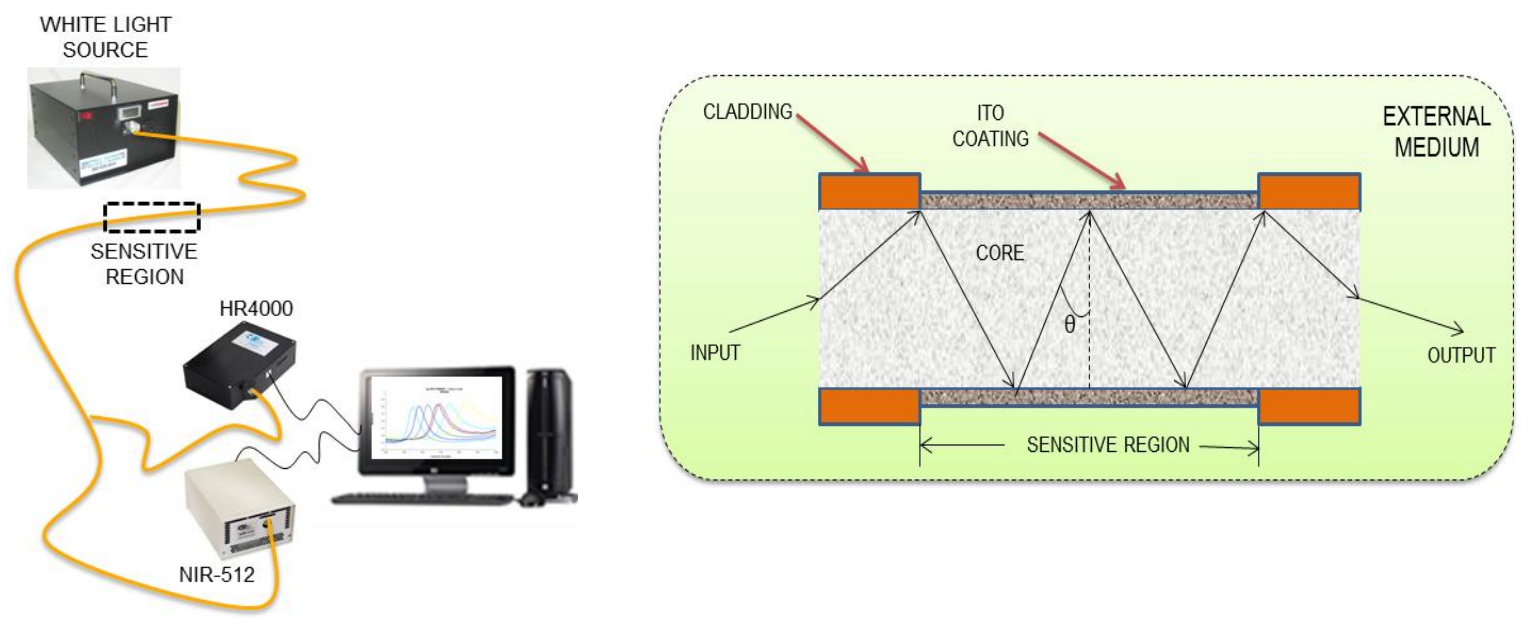

Fig. 2: Left: Experimental setup. Right: Section of the sensitive region. 


\section{Results and discussion}

\section{3.a. Characterization of ITO coatings}

ITO coatings fabricated on optical fibers were characterized using a scanning electron microscope (FESEM, from Carl Zeiss Inc.) and an atomic force microscope (Innova AFM, Veeco Inc.). Figure 3 shows the scanning electron microscope (SEM) image of a cross section of the ITO coated optical fiber. These coatings reveal an homogeneous coverage of the optical fiber core surface. In this case, the coating thickness is approximately $250 \mathrm{~nm}$. Additionally, atomic force microscope (AFM) images of coatings deposited on the fiber were obtained, as it is shown in Fig. 4, with an average roughness RMS of $0.34 \mathrm{~nm}$.

\section{3.b. Characterization of fiber optic refractometers}

The spectral response of the refractometer was obtained for different EMRI, air $(n=1)$, water $(n=1.333)$ and glycerol $80 \%(n=1.447)$. Figure 5 shows the absorption spectra with the maximum absorbance wavelength (resonance wavelength)

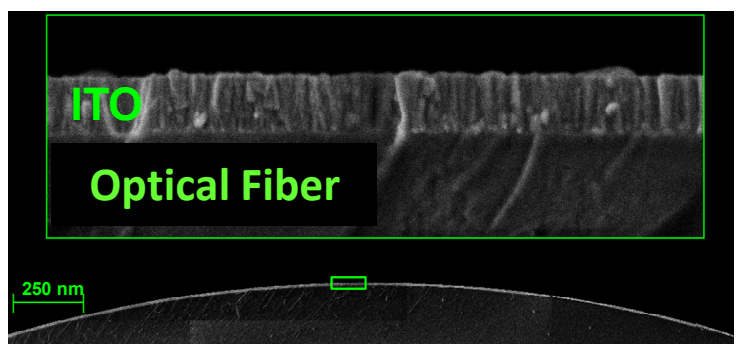

Fig. 3: SEM micrograph of the ITO coating.
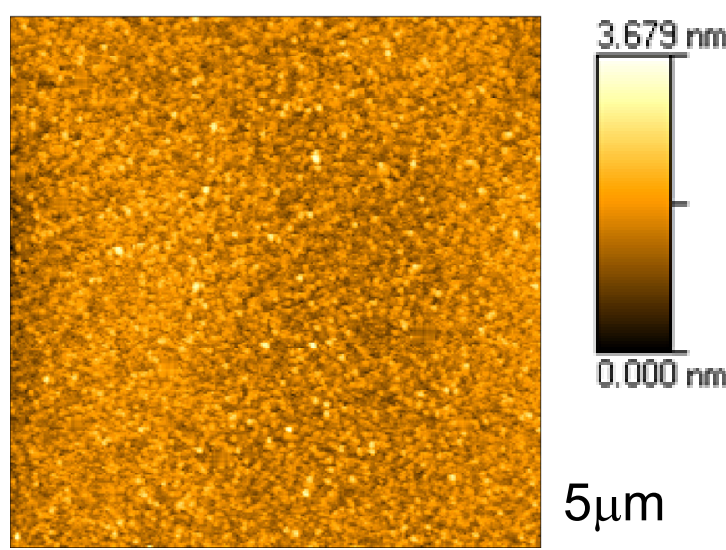

Fig. 4: AFM image of the ITO coating. in the visible and near infrared spectral region. It is also denoted that the resonance wavelength shifts to the red when the EMRI is increased. Therefore, the absorption in the visible area (approximately 380-750 $\mathrm{nm}$ ) shifts as a function of the EMRI. It is also important to remark the differences between the transmission and absorption bands (greater than $10 \mathrm{~dB}$ in all cases), which are denoted by the abrupt slopes. This had not been observed in previous devices based on ITO coatings [1].

The high absorption bands enable to tune the colour of the light at the output of the fiber as a function of the EMRI. Thus, the input colour of the light at the input and at the output of the fiber can be observed by using the setup shown in Fig. 6. When the external medium surrounding the sensitive region is air (see Fig. $6 a$ ), the colour of the light at the output of the fiber is yellow; if the external medium is water the colour becomes orange (see Fig. 6b). However, if the sensitive region is immersed in a solution containing $80 \%$ glycerol, the colour of the light at the end of the fiber becomes pink (see Fig. 6c).

The above results can also be expressed in terms of the chromaticity coordinates $(x, y)$ using the SpecraSuite ${ }^{\circledR}$ color application tool software

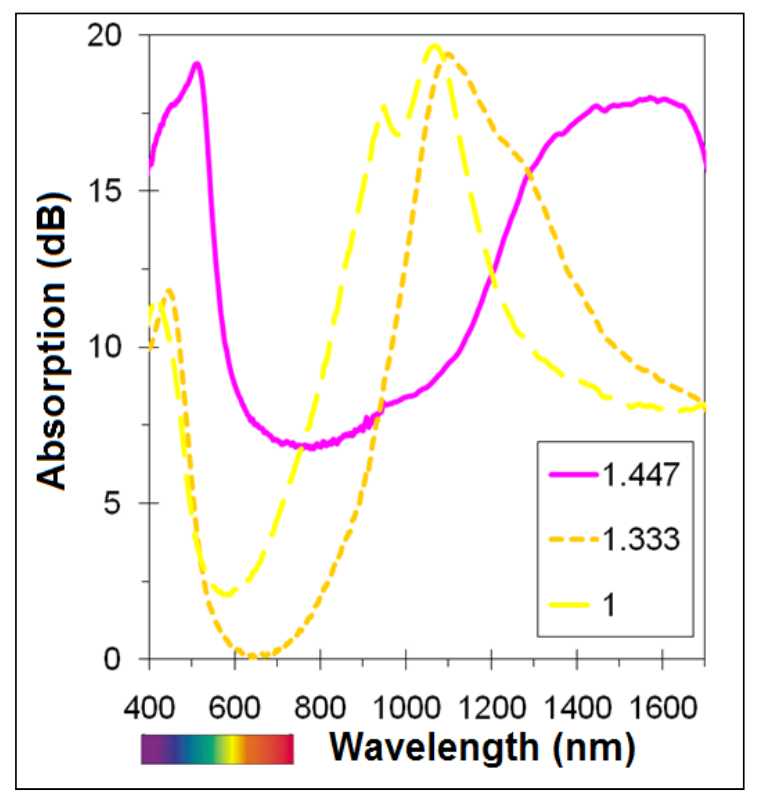

Fig. 5: Spectral Response of optical fiber refractometers for different refractive indices of the external medium. 
WHITE LIGHT SOURCE SENSITIVE REGION
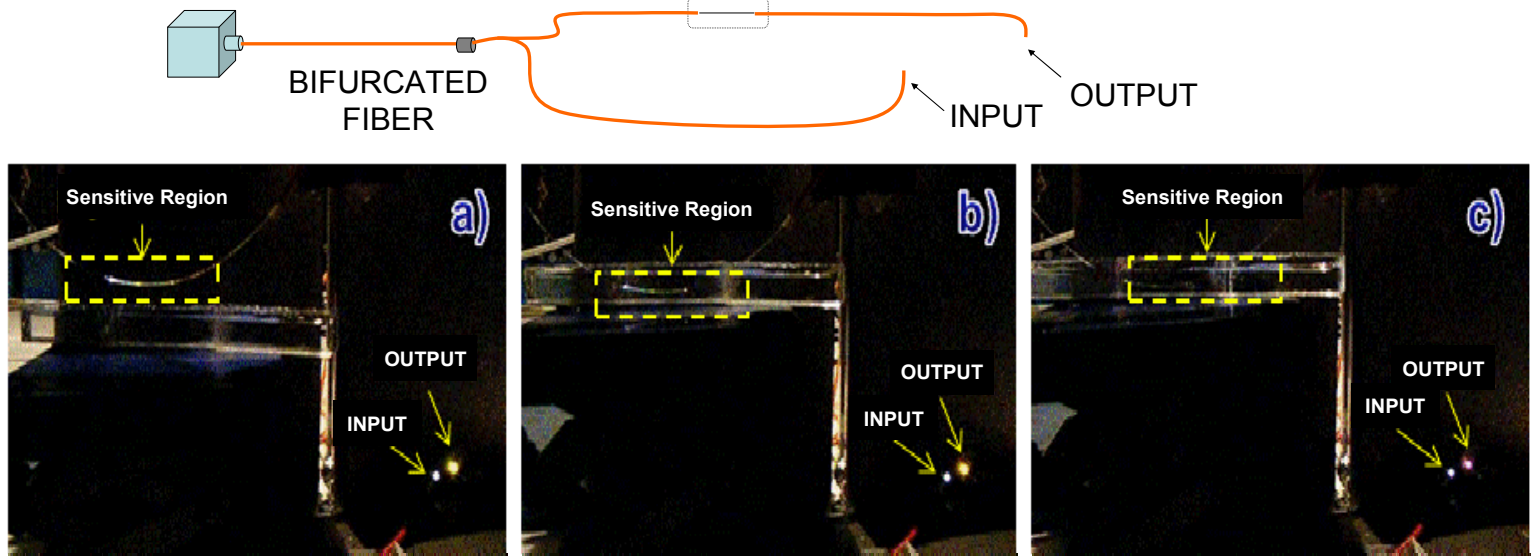

Fig. 6: Top: Schematic representation of the setup used to observe the colour of the light at the input and output of the fiber. Bottom: Colour at the fiber output when the EMRI is a) air $(n=1)$, b) water $(n=1.333)$ and c) $80 \%$ glycerin $(n=1447)$.

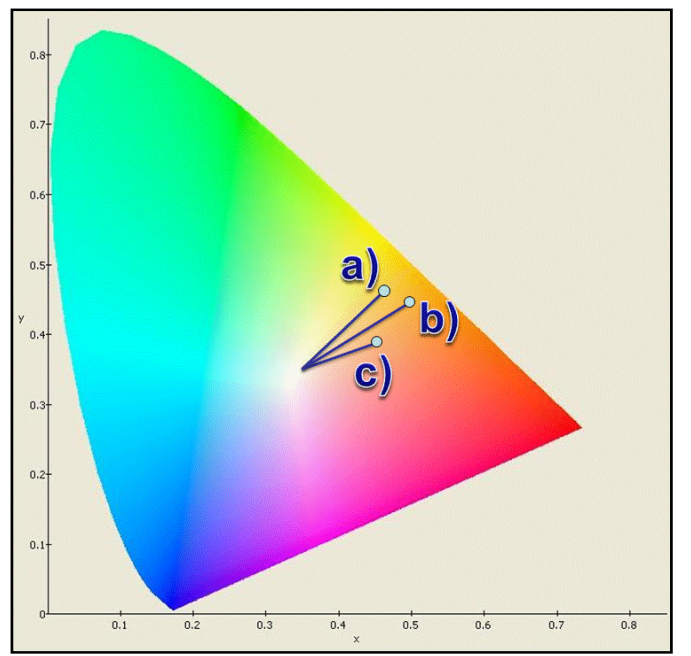

Fig. 7: Chromaticity coordinates of the output signal for different external means: a) air, b) water c) $80 \%$ glycerin.

provided by OceanOptics Inc. Thus, Fig. 7 represents the chromaticity coordinates within the colour chart, which corresponds to the changes in colour produced for each of the different media. In particular, the chromaticity coordinates $(x, y)$ of the light at the output of the fiber are $(0.471,0.463)(0.507,0.445)$ and $(0.445,0.392)$ for air, water and $80 \%$ glycerin respectively. Since the change in colour is relatively small compared with the variation in refractive index it is necessary the measurement of the resonance wavelength as well as the utilization of a high spectral resolution detector is required for a precise refractive index determination. Nevertheless, colour changes have been pointed as an interesting result for a rough determination of refractive index changes within the first and maybe second decimal.

\section{Conclusions}

ITO coatings have been fabricated onto the core of optical fibers using the sputtering technique. Furthermore, it has proven that ITO-coated optical fiber devices can act as refractometers in the visible and near infrared spectrum. Moreover, these resonances show high attenuation bands, which had not been seen before in previous works of our group with ITO coatings fabricated using the dip-coating technique [1]. Owing to its high absorption, these resonances enable to observe changes in the colour of the light at the output of the fiber. This also permits the utilization of these devices as optical filters in the visible spectrum modulated by the external index of refraction surrounding the filter, which is the main aim of this work.

\section{Acknowledgements}

This work was supported by the Spanish Ministry of Science and Innovation TEC201017805. The authors also thank FIDENA SA for the use of SEM. 